\title{
Recursos para el diagnóstico psicopedagógico del TDAH y comorbilidades
}

\author{
Vicente Félix Mateo
}

Investigador del Hospital Clínico de Valencia.

Psicopedagogo Secundaria.

España

psicofelix@hotmail.com 


\section{Resumen}

\section{Introducción:}

El Trastorno por déficit de atención con hiperactividad (TDAH) tiene una conceptualización, prevalencia, etiología, pronóstico y evaluación que los especialistas en educación deben conocer para dar respuesta a las necesidades educativas que plantean estos alumnos. En este artículo se habla de la conceptualización, comorbilidad y evaluación del TDAH.

\section{Palabras Clave:}

Trastorno por Déficit de Atención con Hiperactividad, evaluación psicopedagógica del TDAH. 


\section{1. ¿Qué entendemos por trastornos hiperactivos?}

El trastorno por déficit de atención con hiperactividad (TDAH) se caracteriza como un patrón persistente de inatención, exceso de actividad e impulsividad, resultado tres subtipos básicos según el DSM-IV-TR (APA, 2002): el predominantemente inatento/desorganizado (TDAH-I), con dificultades tanto en la organización y planificación, como en atención sostenida. El subtipo predominantemente hiperactivo-impulsivo, cuya característica neuropsicológica básica sería la dificultad significativa en autorregular su comportamiento, debido a una disfunción en el control inhibitorio, y por último, el subtipo combinado (TDAH-C), es decir, tanto con sintomatología de inatención, y de exceso de actividad e impulsividad, siendo de los tres subtipos el que aparece con más frecuencia.

Tabla 1. Subtipos neuropsicológicos de TDAH en el DSM-IV-TR (2002)

\begin{tabular}{|l|l|l|}
\hline \multicolumn{1}{|c|}{ Inatención } & \multicolumn{1}{c|}{ Hiperactividad } & \multicolumn{1}{c|}{ Impulsividad } \\
\hline $\begin{array}{l}\text { No presta suficiente atención } \\
\text { a los detalles }\end{array}$ & $\begin{array}{l}\text { Mueve en exceso manos y } \\
\text { pies (hiperkinesia) } \\
\begin{array}{l}\text { Tiene dificultades en la } \\
\text { atención sostenida } \\
\text { Abandona su asiento en la } \\
\text { clase. }\end{array}\end{array}$ & $\begin{array}{l}\text { Responde de forma precipi- } \\
\text { tada a las preguntas }\end{array}$ \\
$\begin{array}{l}\text { Parece no escuchar cuando se } \\
\text { le habla directamente }\end{array}$ & $\begin{array}{l}\text { Corre o da saltos en situa- } \\
\text { ciones inapropiadas } \\
\text { Dificultad para finalizar las } \\
\text { tareas (no oposicionismo) }\end{array}$ & $\begin{array}{l}\text { Tiene dificultad para realizar } \\
\text { actividades tranquilas } \\
\text { guardar el turno en una fila }\end{array}$ \\
$\begin{array}{l}\text { Dificultad para organizar sus } \\
\text { actividades o tareas }\end{array}$ & $\begin{array}{l}\text { Interrumpe o se inmiscuye } \\
\text { en actividades de otros }\end{array}$ \\
$\begin{array}{l}\text { Rechaza cualquier esfuerzo exceso } \\
\text { mental sostenido }\end{array}$ & $\begin{array}{l}\text { Actúa como si estuviese } \\
\text { impulsado "por un motor". }\end{array}$ & \\
$\begin{array}{l}\text { Pierde cosas necesarias } \\
\text { Se distrae por estímulos } \\
\text { irrelevantes }\end{array}$ & \\
Es olvidadizo & & \\
\hline
\end{tabular}

Para el diagnóstico de TDAH, la sintomatología debe haber aparecido antes de los 7 años, y persistido durante al menos 6 meses con una intensidad desadaptativa en relación con 
el nivel de desarrollo del niño (APA, 2002). Igualmente, debe existir concordancia en al menos dos contextos distintos (ej. casa y escuela) para su diagnóstico.

La prevalencia del trastorno se sitúa entre el 3-6\%. En un estudio realizado recientemente en una muestra extraída entre 6 colegios públicos y 1 concertado, la incidencia fue del 3\%.(ver Félix, 2003). Dicho de otra forma, cada 4 aulas de nuestros centros, encontraremos al menos un alumno con TDAH. Pese a ser uno de los trastornos con inicio en la infancia y adolescencia más diagnosticados en las consultas de médicos, psicólogos, neuropediatras y psiquiatras infantiles, existen enormes dificultades desde el ámbito de la educativo para su diagnóstico e intervención, siendo necesaria una formación más específica para los profesionales de la educación, que diariamente se enfrentan a ellos, teniendo además que transmitirles una serie de conocimientos, actitudes, aptitudes y normas que les permita desenvolverse como personas responsables una vez abandonen el sistema educativo.

Un aspecto esencial tanto en la detección de necesidades como en la respuesta a las mismas, es el trabajo multidisciplinar con el que se tiene que abordar este trastorno. La relevancia del contexto escolar en general, y de los profesionales de la educación en particular, es obvia, ya que el niño hiperactivo pasa gran parte de su tiempo en este contexto, y es donde normalmente aparecen los síntomas de una forma clara. Pero igualmente importante resulta el contexto hospitalario, donde se proporcionará al niño una respuesta farmacológica, en los casos necesarios, que ayudará a paliar la sintomatología básica, a la par que permitirá una mejor reeducación desde la escuela. En este sentido, existe un trabajo interesante realizado por un psicopedagogo / neuropsicólogo y neuropediatras (ver Andrés, Castelló, Félix y Casti1lo, 2005).

Por lo que respecta al trabajo psicopedagógico con estos alumnos, es necesario clarificar en la medida de lo posible hasta qué punto la sintomatología de un alumno hace referencia a un hipotético TDAH, o por el contrario, se enfrenta ante un niño el que es necesario realizar un diagnóstico diferencial con otro tipo de trastornos que comparten sintomatología común o similar (como con el trastorno de conducta). Por otra parte, el grado de comorbilidad del TDAH con otros trastornos (ansiedad, depresión, Tourette, dificultades de aprendizaje, trastorno de conducta, trastorno obsesivo-compulsivo, trastorno oposicionista-desafiante, cefaleas, tics, baja competencia social, epilepsia, etc.) es muy elevado (ver Roselló, Amado y Bo, 2000). Por ello, es importante delimitar qué trastorno/s aparece/n de forma comórbida al 
TDAH, de cara a planificar una intervención tanto psicopedagógica como farmacológica más ajustada a sus necesidades.

\section{Identificación y evaluación de las necesidades}

La evaluación del TDAH es un aspecto complejo. Obviamente, el proceso de evaluación no es siempre igual. Existen niños que ofrecen una frecuencia y severidad tan elevada de la sintomatología, que simplemente con la observación directa y la cumplimentación de una serie de cuestionarios garantizan prácticamente un diagnóstico acertado. Sin embargo, muchas otras veces no tenemos en frente al "hiperactivo de libro". Puede que aparezcan algunos síntomas, o tal vez tengamos que indagar sobre otros aspectos que aparentemente se muestran latentes. Así, ante el comentario de un tutor al psicopedagogo sobre un alumno que tiene un bajo nivel académico, no termina nada que empieza y casi siempre suele estar distraído, podría entrar en el perfil de un niño desatento, pero también este perfil puede observarse en un alto porcentaje de alumnos de la educación secundaria obligatoria que no tienen un TDAH. Otras veces, la información de los contextos naturales en los que el niño se desenvuelve es contradictoria o parece que estén describiendo a niños distintos. Por ejemplo, puede aparecer un perfil en el que desde el contexto familiar muestren a su hijo como un niño muy movido, aunque la severidad de los síntomas no alcance el mínimo suficiente y sin dificultades atencionales, mientras que en el contexto escolar aparezca como un niño inquieto, algo impulsivo, pero sobre todo con muchos problemas en el control de la atención sostenida. También puede ocurrir que el nivel socio-cultural de los padres sea muy bajo, y por lo tanto tengamos dificultades para que cumplimenten las escalas comportamentales. Un último ejemplo podríamos encontrarlo ante un profesor que busca constantemente orden y silencio absoluto entre sus alumnos, e informa de un alumno que se mueve y habla demasiado (aunque puede que no sea hiperactivo) o por el contrario un profesor excesivamente tolerante que ante los comportamientos disruptivos tiende a minimizar el problema del niño (“es algo movido, pero ya irá madurando..."). Estos son sólo algunos ejemplos de la casuística que nos podemos encontrar en los centros educativos ante las demandas de valoración sobre TDAH.

\section{Protocolo de identificación}

1. En primer lugar, es aconsejable que cuando el tutor nos deriva al alumno, nos aporte alguna información relevante en torno a su comportamiento dentro del aula (si es dis- 
ruptivo -habla cuando no debe, se mueve en exceso, llama constantemente la atención-; si tiene dificultades para mantener la atención -se despista con cualquier estímulo irrelevante, se cansa rápido, no termina sus tareas

2. Bien a través de los padres, del tutor, o de ambos, deberíamos indagar en torno a la historia evolutiva y clínica del niño. Aspectos sobre el. desarrollo prenatal y perinatal (si hubo abuso de ansiolíticos, antidepresivos o sustancias psicotrópicas, si hay en la familia algún miembro con TDAH, si fue un embarazo de riesgo, peso del niño al nacimiento, escala Apgar, etc.), adquisición de los distintos hitos evolutivos (control céfalo-caudal, lenguaje, desarrollo motor, control de esfínteres, etc.). Asimismo, es interesante conocer la historia escolar del niño. Desde la educación infantil (adaptación, relaciones interpersonales, si hubo excesivo movimiento, desapego de los padres, etc.). Respecto a la Educación Primaria, nos interesa conocer cómo adquirió la lectoescritura, cómo es su nivel de atención dentro del aula, si hay que estar constantemente supervisando su trabajo, si hay que llamarle muchas veces la atención para que esté sentado, si hay que aplicar correctivos, cuáles y con qué frecuencia, cómo es la relación con sus compañeros/as de clase, etc.).

3. Observación directa. Es necesario analizar el comportamiento del niño dentro del aula, ya que nos proporcionará claves inmediatas respecto a qué comportamiento/s apare$\mathrm{ce} / \mathrm{n}$ de forma más frecuente y su severidad. Para ello, resulta útil realizar un seguimiento a través de registros observacionales en distintos momentos del día y en distintos días.

4. Pase de pruebas. Se analizan extensamente en el siguiente punto. Es importante advertir que las pruebas harán referencia a distintos dominios: comportamental, social y de funcionamiento ejecutivo.

\section{Evaluación psicopedagógica del tdah}

\subsection{Estimaciones comportamentales para padres y profesores:}

\section{a) Escala Conners (padres y profesores)}

El Cuestionario de Hiperactividad de Conners (1973) es el instrumento más usado en la actualidad en las investigaciones sobre los trastornos por déficit de aten- 
ción con hiperactividad. El cuestionario está integrado por 10 ítems de fácil redacción que describen conductas relevantes del trastorno hiperactivo. Cada ítem oferta cuatro alternativas de respuesta en función de la frecuencia de ocurrencia de la conducta mencionada. Los profesores deben poner una cruz en la casilla correspondiente, que puede ser "siempre", "muchas veces", "a veces" o "nunca". Su cumplimentación es muy simple y se puede realizar en pocos minutos.

\section{b) Escala IOWA de Hiperactividad y Agresividad de Conners para profesores}

Este Cuestionario es el resultado del estudio realizado por Loney y Milich (1982) sobre el cuestionario de Conners (1973). Los autores encontraron dos factores relativamente independientes, cuyos ítems más representativos pasaron a formar las 2 subescalas, con 5 ítems cada una que componen este cuestionario: 1) InatenciónHiperactividad y 2) Agresividad-Oposicionismo. Ambas escalas están, por tanto incluidas en el test de Conners (versión extensa). La escala "inatención-hiperactividad" está formada por los ítems 1, 2, 4, 8 y 9 del Test del Conners abreviado arriba mencionado. Se valora de la misma manera que éste, con lo que el rango de puntuaciones posible oscila entre 0 y 15 . El punto de corte mínimo para considerar que un niño de segundo ciclo de Primaria obtiene altas puntuaciones en este factor es de 9. La escala de Oposicionismo estaría incluida en los ítems del 11-15.

\section{c) Listado de síntomas del DSM-IV-TR}

El Cuestionario para padres y profesores DSM-IV (APA, 1994, 2002), es una prueba que recoge los criterios diagnósticos propuestos por el A.P.A en la cuarta edición de su manual diagnóstico (1994) y en la edición revisada (2002) para la categoría de Trastorno por Déficit Atencional con Hiperactividad. Los nueve primeros ítems recogen aspectos relacionados con la Inatención-Desorganización y los nueve siguientes se dirigen a evaluar la Hiperactividad-Impulsividad (del ítem 10 al 15 "Actividad"; del ítem 16 al 18 "Impulsividad"). Existen cuatro alternativas de respuesta ("nunca", "algunas veces", "bastantes veces" y "muchas veces") y se considera que un niño cumple los criterios del TDAH, subtipo combinado, si es valorado por su maestro/a y por sus padres en las alternativas de respuesta "bastantes veces" o "muchas veces" en al menos 6 ítems de cada una de las dos partes de esta prueba. 


\section{d) Cuestionario de Situaciones en la Escuela. (Barkley, 1981).}

La información que suministra este cuestionario permite realizar la imprescindible conexión entre los deficits atencionales de un niño y las situaciones en las que estas deficiencias provocan más problemas: trabajo independiente, actividades en grupo, etc. Por otra parte, este instrumento puede ayudar al clínico a comprender los comportamientos del profesor que, en situaciones concretas, minimizan o intesifican el impacto negativo de los problemas atencionales de un estudiante.

\section{e) Cuestionario de Situaciones en el Hogar (Barkley, 1981).}

Su planteamiento y objetivos es similar al Cuestionario dirigido a la escuela, pero en este caso las situaciones que se recogen hacen referencia al contexto del hogar, por ejemplo, llamadas telefónicas, cuando se reciben visitas en casa, durante las comidas, etc.

\section{f) Escalas para la evaluación del déficit de atención e hiperactividad (E.D.A.H). (Farré y Narvona, 1997)}

La finalidad del E.D.A.H. es recoger información sobre la conducta habitual del niño. Para ello ofrece un método estructurado de observación para el profesor, compuesto por 20 elementos, que después de ser analizados, se desglosan en una escala global y cuatro tipificadas.

\section{g) Inventario de síntomas para niños y adolescentes Stony Brook (Gadow y Spraf- kin, 1995)}

Este cuestionario está dirigido a realizar una evaluación inicial de síntomas conductuales, afectivos y cognitivos de psicopatologías de la infancia y de la adolescencia. Los ítems están basados en el Manual Diagnóstico y de los Trastornos Mentales de la APA (DSM-IV, 1994) y permiten al psicopedagogo recoger información de los padres y/o profesores relativa a psicopatologías. El inventario recoge información en torno a los siguientes trastornos: Trastorno negativista/desafiante; Trastorno disocial; Trastorno de Tourette; Trastornos de Ansiedad (ansiedad generalizada, fobia social, trastorno obsesivo-compulsivo, trastorno de estrés postraumático); Trastornos del estado de ánimo (depresión mayor, trastorno distímico); Trastorno de ansiedad por separación; Enuresis y Encopresis. 


\subsection{Tests y pruebas específicas para la evaluación de la atención}

\section{a) Test de Caras (Thurstone y Yela, 1979)}

El test de percepción de diferencias (Caras) evalúa las aptitudes perceptivas y de atención. La prueba está formada por 60 elementos gráficos que representan dibujos esquemáticos con trazos elementales. Es una prueba de aspecto lúdico y simple adecuado para poblaciones de bajo nivel cultural y demasiado elemental para niveles culturales superiores. El tiempo de pase de la prueba es de tres minutos, y aporta información respecto a la atención sostenida del niño.

b) Test de cancelación de figuras (Rudel, Denkla y Borman, 1978)

Esta prueba consta de tares sencillas dirigidas a valorar la persistencia visual de niños entre los 4 y los 13 años. En el subtest de Rombos el niño debe localizar y señalar todos los rombos que aparecen aleatoriamente entre 140 formas geométricas. E1 subtest 592 exige que el niño señale el número 592 entre un total de 140 números de tres dígitos. En ambos subtests se contabiliza el número total de errores de omisión y de comisión, y el tiempo total de ejecución de la tarea.

\section{c) Test de ejecución continua (CPT; Rosvold et al., 1956)}

Esta tarea presenta sucesivamente 500 letras durante $400 \mathrm{mseg}$. con un intervalo entre estímulo de $600 \mathrm{mseg}$. (tiempo total de la tarea 8 minutos). El sujeto debe responder cada vez que aparece un X precedida por una A. Eso ocurre en 50 ocasiones, pero en otras 50 veces aparece la X no precedida por A y otras 50 ocasiones las A no seguida por X. Las medidas de impulsividad son los errores de omisión (número de AX no detectadas) y los de comisión (número de veces que se responde en ausencia de una $\mathrm{X}$ precedida por una $\mathrm{A}$ ). Es una tarea clásica en la evaluación de la atención, utilizada frecuentemente en investigación (ver Félix, 2002, 2003; Ávila, Cuenca, Félix et al., 2004)

\section{d) Perfil atencional del WISC-R (Wechsler, 1974)}

El factor atencional de la Escala de Inteligencia para niños de Weschler está compuesto por varias subescalas: Aritmética, Dígitos y Claves. El subtest de Aritmética está dirigido a valorar la atención dividida (habilidad del niño para atender a dos fuentes de información distintas. El subtest de Dígitos evalúa la vigilancia (predisposi- 
ción del sujeto para emitir una respuesta ante un estímulo y mantenerla a lo largo del tiempo). Por último, el subtest de Claves mide la atención sostenida (capacidad para mantener la concentración a lo largo del tiempo)

\section{e) Pruebas de Atención del Seminario de Pedagogía del Instituto Pontificio S. Pío $X(1961)$}

Puede aplicarse a chicos entre los 11 y 16 años en un tiempo de 20 minutos. La prueba está compuesta por tres subtests: Cuadrados, Laberintos y Letras. El test de cuadrados exige atención sostenida en una tarea de discriminación perceptiva compleja. El test de Laberintos valora la capacidad atencional ante una tarea de rastreo y control ocular, mientras que el test de Letras guarda una gran similitud con los planteamientos de los tests de Ejecución continua que son las pruebas más utilizadas para evaluar atención sostenida

\section{f) Subtest de Integración Visual del ITPA (Test Illinois de Aptitudes Psicolinguísti- cas. Kirk, McCarthy y Kirk, 1986 ).}

Para niños con edades comprendidas entre los 2 y 10 años de edad. El niño tiene que identificar objetos familiares (peces, botellas, martillo-serruchos y perros) que aparecen visibles en parte y que están inmersos en un fondo distractorio. Se trata de una tarea compleja por lo que, además de evaluar atención sostenida, constituye una medida de la capacidad de reflexión y de perseverancia.

\subsection{Pruebas de impulsividad}

a) MFFT (Servera y Llabrés, 2000).

Consta de 4 ensayos de práctica y 16 ensayos de la prueba en los que aparece una figura superior, y seis inferiores entre las que hay cinco parecidas y una idéntica. En cada ensayo, el participante debe indicar con el ratón que figura de las inferiores es idéntica a la superior. En caso de error, se debe repetir la respuesta hasta acertar. Se registra para cada ensayo el número de errores y la latencia de la primera respuesta. De estas dos medidas, se derivan los índices de impulsividad (restando los valores estandarizados de errores y latencia) y eficacia (sumando los valores estandarizados de errores y latencia; ver Servera y Llabrés, 2000, para más detalles). 


\section{b) Test de Ejecución Continua CPT (Errores de comisión)}

El CPT permite, a través de los errores de comisión (pulsar la barra espaciadora cuando no aparece el objetivo diana) medir la impulsividad del niño. Existen estudios que han encontrado una fuerte correlación entre los errores de comisión y las estimaciones comportamentales de impulsividad de padres y profesores (ver Félix, 2002, 2003).

\subsection{Pruebas de control inhibitorio}

a) Test de Stroop (Stroop, 1935)

El "Efecto Stroop"consiste en un error o retraso provocado al nombrar un determinado color cuando éste se presenta al sujeto como el color en que esta escrita una palabra, que a su vez muestra otro color incongruente con el anteriormente expuesto. Se ha comprobado que las dificultades para leer palabras pueden ser debidas a lesiones en le hemisferio izquierdo, mientras que la identificación de colores se relaciona con el hemisferio derecho. Tiene la ventaja de que su uso no depende del nivel cultural del sujeto y que sus estímulos son sencillos además de administrarse rápidamente.

La versión española adaptada de Golden (1978), consta de tres partes que se realizan separadamente durante 45 segundos cada una. La primera condición de PALABRAS consiste en leer las palabras ROJO, VERDE y AZUL escritas en negro sobre fondo blanco. La segunda condición de COLORES consiste en nombrar el color de XXXXX que puede ser rojo, verde o azul. En la tercera condición de COLORPALABRAS aparecen las palabras ROJO, VERDE y AZUL escritas en colores diferentes. En cada condición, los participantes deben emitir tantas respuestas correctas como sea posible en $45 \mathrm{seg}$. La variable que mide impulsividad es la interferencia, que se calcula restando el número de respuestas en la condición de COLOR-PALABRAS del de la condición COLORES.

\section{b) Sun-Moon Stroop (Gerstadt, Hong y Diamond, 1993)}

Se presentan al niño 16 tarjetas en las que aparece en una secuencia cuasialeatoria el sol y la luna. Cuando se le presenta el sol, el niño debe decir "noche", y cuando se le presenta la luna, debe decir “día”. Esta versión del Test de Stroop resulta especialmente útil con niños en edad preescolar, ya que no requiere tener adquiridas las habilidades de lecto-escritura, produciéndose igualmente la interferencia (decir día ante una luna o noche ante un sol). 


\section{c) Tarea de Stop (Logan y Cowan, 1984)}

Esta tarea supone una adaptación de la utilizada por Avila y Parcet (2001), siendo apta para su uso en la evaluación de niños con TDAH (ver Ávila, Cuenca, Félix et al., 2004; Félix, 2002, 2003; Miranda, Félix y Ávila, en prensa). Se basa en la ejecución de una doble tarea concurrente: la principal y la de stop. La tarea principal consiste en determinar lo más rápidamente posible si un estímulo es una $\mathrm{X}$ o una $\mathrm{O}$ respondiendo en dos teclas diferentes del ordenador. La tarea tiene 280 ensayos. Tras la presentación de la $\mathrm{X}$ o la $\mathrm{O}$, y por encima de ellas, se presenta algunas veces un círculo verde (la señal de stop) durante $150 \mathrm{mseg}$. que indica al sujeto que debe intentar no ejecutar la respuesta de la tarea principal. Siguiendo el procedimiento usado por Logan, Schachar y Tannock (1997), la señal de stop se presentaba inicialmente $250 \mathrm{~ms}$ después del estímulo de la tarea principal, para ir ajustándose posteriormente en función de la ejecución: si el participante consigue inhibir la respuesta, la señal de stop se presentará $50 \mathrm{mseg}$. más tarde, mientras que si no lo consigue se presentará $50 \mathrm{mseg}$. antes. De esta manera se persigue encontrar el momento en el que el participante consigue inhibir la respuesta en un 50\% de ocasiones, ajustándose la tarea al niño (y no viceversa).

\section{d) Tarea del círculo}

Esta tarea fue adaptada de la utilizada por Bachorowski y Newman (1990). Los participantes fueron instruidos para trazar con el dedo índice de la mano preferente $3 / 4$ partes de círculo de $24.3 \mathrm{~cm}$. de diámetro tan lento como sea posible. El círculo estaba dibujado sobre papel y tenía marcados el inicio y el final. La variable utilizada para evaluar la impulsividad era el tiempo de trazado. Inicialmente se realizó un ensayo de práctica.

\subsection{Pruebas de organización y planificación}

\section{a) Test Trail-Making}

Esta tarea tiene dos partes. En la Forma A, los participantes recibían una hoja papel con los números de 1 al 25 situados aleatoriamente, y la tarea consistía en trazar una línea uniéndolos en orden consecutivo, sin levantar el lápiz del papel. En la Forma $\mathrm{B}$, aparecían los números del 1 a al 13 y las 12 primeras letras del abecedario, y la tarea consistía en unir alternativamente letras y números consecutivos (ej. 1A2B3C4D). 
La medida derivada relacionada con la impulsividad es la interferencia medida a partir de la diferencia en tiempo (o en errores) entre las formas A y B.

\section{b) Figura compleja de Rey (Rey, 1994)}

Test guestáltico que evalúa la actividad perceptiva y la memoria visual. Muy usado con niños y aprecia el nivel de desarrollo intelectual y perceptivo-motor, atención, memoria visual inmediata, esfuerzo de memorización y rapidez de funcionamiento mental.

\section{c) Torre de Londres (Shallice, 1982)}

Diseñada inicialmente para identificar alteraciones en los procesos de planificación en pacientes frontales. Consiste en presentar al niño 3 bolas de distintos colores que se pueden colocar en tres palos de distintas alturas. El niño tiene que poner las bolas que se presentan inicialmente en otro palo, pero en la misma posición, y sólo utilizando un número determinado de movimientos y cumpliendo una serie de reglas. El nivel de complejidad del test se manipula a partir del incremento mínimo de movimientos. Exige planificación en torno a una serie de metas futuras que permita resolver los problemas.

\subsection{Pruebas de memoria de trabajo}

\section{a) Prueba de memoria de conteo (Case, Kurland y Goldberg, 1982)}

Esta tarea requiere que el niño procese información visual on-line y almacene dicha información para ser recobrada posteriormente. Se presenta un juego de tarjetas con puntos amarillos y azules, distribuidos en forma aleatoria. Una vez que el niño ha contado el número de puntos amarillos en cada tarjeta que compone el juego, el niño debe repetir, en orden, el número de puntos que aparecen en cada tarjeta. El niño debe repetir el procedimiento, tres veces consecutivas, en los cinco niveles formados por 2 , 3, 4, 5, o 6 tarjetas cada uno. Así, la tarea comprende 15 ensayos que van aumentando en nivel de dificultad. No se proporciona retroalimentación durante la administración, pero la tarea se interrumpe si el niño ha fallado en todos los ítems de un mismo nivel.

\section{b) Tarea de memoria de trabajo de oraciones (Siegel y Ryan, 1989)}

Es una tarea de memoria de trabajo verbal. Comprende 42 oraciones, a las que les falta la última palabra. Estas oraciones están distribuidas en grupos de 3 oraciones 
cada grupo. Cuando se lee la primera frase, el niño debe decir la palabra que falta. El mismo proceso se repite con la segunda y tercera frases. Al final de cada bloque, debe decir las tres últimas palabras de cada frase, repitiéndolas en el mismo orden.

\section{c) Tarea de recuerdo espacio-temporal (Dubois et al., 1995)}

Es una tarea computerizada que valora la memoria de trabajo viso-espacial. Está compuesta por 30 ensayos; en cada uno de ellos se presentan al 12 cuadrados de color azul, teniendo el niño que estar atento mirando los cuadrados que, de forma sucesiva, cambiaban de color (de azul a rojo). A continuación, la pantalla se obscurece y vuelven a aparecer los 12 cuadrados azules. El niño debe reproducir la secuencia que se había producido en el cambio de cuadrados de azul a rojo. La demanda de memoria de trabajo viso-espacial se produce porque se manipula el tiempo que la pantalla neutra (negra), periodo tras el cual el niño reproduce la secuencia.

\subsection{Perseveración en la respuesta (mantenimiento del set) a) Test de Clasificación de Cartas de Wisconsin (Heaton, 1981)}

Este test consta de 64 cartas con figuras que varían en la forma (triángulo, cuadrado, redonda o cruz), el color (rojo, azul, verde o amarillo) y el número (uno, dos, tres o cuatro). Los participantes deben aprender en cada momento las reglas del juego, de tal manera que sea capaz de emparejar la carta presente con una de las cuatro posibilidades que aparecen en la parte superior de la pantalla. Las reglas dependen sucesivamente del color, la forma y el número con un total de 6 series. El cambio de regla se realiza cuando se realizan 10 respuestas consecutivas seguidas. La prueba finaliza cuando el sujeto completa las 6 series o categorías, o cuando el sujeto completa 128 ensayos. Esta prueba produce diversas variables relevantes como son el número de aciertos, el número de categorías conseguidas, el número de errores perseverativos (perseverar en la categoría anterior) y el número de errores no perseverativos. En definitiva, podemos decir que evalúa pensamiento abstracto y perseveración en la respuesta.

\subsection{Comorbilidad}

\section{a) Listado de síntomas de comorbilidad Stony Brook (Gadow y Sprafkin, 1995)}

Ya hemos comentado anteriormente que este cuestionario, basado en los listados del DSM-IV, permite realizar de una forma relativamente sucinta un barrido por 
los distintos trastornos comórbidos que con más frecuencia aparecen ligados al TDAH. Puede ser cumplimentado por padres, profesores o por ambos.

b) Ansiedad: CAS (Gillis, J.S.), Listado de síntomas del DSM-IV; entrevista con padres y profesores.

El test CAS permite una apreciación del nivel de ansiedad infantil. Este instrumento permite efectuar una rigurosa exploración psicológica de los procesos de ansiedad en niños de los primeros cursos escolares.

c) Dificultades de aprendizaje: Lecto-escritura (T.A.L.E.; PROLEC; NAC lengua)

- Cálculo (Prueba de Canals, TEA; NAC matemáticas);

Las dificultades de aprendizaje es el trastorno comórbido que antes y con más frecuencia aparece ligado al TDAH, derivado principalmente de las dificultades en la atención. La forma de valorar las dificultades de aprendizaje son múltiples. Aquí planteamos una serie de pruebas estandarizadas, para el análisis de la lecto-escritura y del cálculo. Resulta especialmente útil conocer el nivel de competencia curricular que presenta el alumno, por lo que la información del tutor/a como del profesor/a de pedagogía terapéutica.

Para el análisis de la lectura existen dos pruebas muy utilizadas. Por un lado, la prueba de Toro y Cervera (1980) de análisis de la lecto-escritura, actualizada al TALE 2000. Por otra parte el PROLEC (Cuetos, Rodríguez y Ruano (1996), que evalúa la capacidad global lectora y la comprensión, así como los procesos que intervienen en la comprensión del material escrito, los procesos sintácticos y semánticos.

Por último, para la evaluación del cálculo, existen pocas tareas estandarizadas en el mercado, resultando útil la batería psicopedagógica de CANALS (TEA). También resulta igualmente útil el nivel de competencia curricular en el área instrumental de matemáticas que nos puede proporcionar el tutor.

d) Depresión (Kovacs, M. (1992). Inventario de depresión infantil CDI; Entrevista con padres y profesores; Listado de síntomas del DSM-IV)

El CDI es quizá el instrumento más utilizado en depresión infantil. Puede aplicarse en el contexto escolar para realizar un screening o como elemento diagnóstico. 
No cabe desestimar la observación directa, así como las entrevistas con padres y profesores sobre su comportamiento diario.

e) Comportamiento oposicionista/desafiante (Test de Conners); Trastorno de conducta (observación directa, entrevista padres y profesores, listado DSM-IV), etc.

Es otro de los trastornos que aparecen frecuentemente ligados al TDAH, especialmente al subtipo hiperactivo-impulsivo (TDAH-HI) así como al combinado (TDAH-C). Ya comentamos anteriormente esta escala.

\section{Discusión}

La evaluación psicopedagógica del TDAH resulta un aspecto complejo, no sólo por la exigencia que dicho trastorno exige en torno a su conocimiento para la detección, sino también por la interdisciplinariedad con la que debería abordarse, y que por desgracia, muchas veces resulta inviable.

Pese a ser un trastorno con más de un siglo de existencia (Fue Still en 1904 quien reporta en la literatura científica el primer caso), todavía desconocemos las causas del mismo (aunque sepamos que existen algunos genes implicados como el DAT-1 y el DRD4). En esta línea, las pruebas neuropsicológicas existentes todavía no ofrecen una fiabilidad del 100\%, aunque hay estudios que muestran buenos indicadores (ver Miranda, Félix y Ávila, en prensa).

Es importante valorar el TDAH desde todas las dimensiones (social, cognitiva, comportamental, etc.), además de indagar sobre los posibles trastornos comórbidos que aparezcan, para así poder planificar una intervención más ajustada (tanto psicopedagógica como farmacológica).

La participación de la familia durante el proceso de evaluación y especialmente en la intervención resulta absolutamente necesaria. Será por ello importante implicarles durante todo el proceso, proporcionarles información en torno al trastorno, su conceptualización, su etiología, diagnóstico y pronóstico, así como trabajar las expectativas. 
Por lo que respecta al profesorado, va a tener igualmente un papel preponderante el la evaluación y tratamiento. Desde el contexto escolar obtendremos información significativa en torno a los aspectos sociales (especialmente de interrelación), académicos (nivel de competencia curricular, estilos de aprendizaje, nivel de atención, comportamiento/s disruptivos/s en el aula, etc.).

Por último, resaltar que es necesario derivar a todos los niños susceptibles de padecer un TDAH al servicio de neuropediatría del hospital más cercano, con la finalidad de que estimen la conveniencia de combinar, junto con la intervención psicopedagógica, tratamiento farmacológico. Hay que resaltar que además del tratamiento de elección para el TDAH con psicoestimulante (normalmente metilfenidato), existen otros tratamientos farmacológicos para estos niños (bien porque la respuesta al metilfenidato no es la esperable, bien por otros trastornos comórbidos que hacen necesario implementar otra terapia farmacológica que dé una respuesta más precisa a las necesidades del paciente). 


\section{Referencias}

American Psychiatric Association. (2002). Manual Diagnóstico y Estadístico de los Trastornos Mentales. DSM-IV-TR. Barcelona: Masson.

Andrés, M., Castelló, M.L., Félix, V. y Castillo, S. (2005). Valoración del TDAH en una consulta de neuropediatría: Aspectos clínicos y neuropsicológicos. Comunicación presentada en la Sociedad Valenciana de Pediatría. Castellón, junio de 2005.

Ávila, C. y Parcet, M.A. (2001). Personality and inhibitory déficits in the stop-signal task: the mediating role of Gray's anxiety and impulsivity. Personality and Individual Differences, 31, 975986.

Ávila, C., Cuenca, C., Félix, V., Parcet, M.A. y Miranda, A. (2004). Measuring impulsivity in schoolaged boys and examining its relationship with ADHD and ODD ratings. Journal of Abnormal Child Psychology, 32, 295-304.

Bachorowski, J.A. y Newman, J.P. (1990). Impulsive motor behavior: effects of personality and goal salience. Journal of Personality and Social Psychology, 58, 512-518.

Barkley, R.A. (1981). Hyperactive Children: A Handbook of Diagnosis and Treatment. New York, Guilford Press.

Case, R., Kurland, M. y Goldberg, J. (1982). Operational efficiency and the growth of the short term memory. Journal of Experimental Child Psychology, 33, 386-404.

Conners, C.K. (1973). Rating scales for use in drug studies with children (Especial issue). Psychopharmacological Bulletin, 24-84.

Cuetos, F., Rodríguez, B. y Ruano, E. (1996). PROLEC. Batería de evalación de los procesos lectores de los niños de educación primaria.

Farré, A. y Narbona, J. (1997). Escalas para la evaluación del trastorno por déficit de atención con hiperactividad. Madrid: TEA Ediciones.

Félix, V. (2002). Evaluación del control inhibitorio mediante la tarea de stop. Tesina. Universidad de Valencia.

Félix, V. (2003). Evaluación de la impulsividad en el trastorno por déficit de atención con hiperactividad a través de tareas neuropsicológicas. Tesis doctoral. Universidad de Valencia.

Gadow, K. y Sprafkin, J. (1995). Child Symptom Inventories. The revised CSI Parent /teacher Checklist. Checkmate Plus, LTD. Stony Brook, NY.

Gerstadt, C., Hong, Y. y Diamond, A. (1994). The relationship between cognition and action: performance of children 3 1/2 - 7 years old on a Stroop-like day-night test. Cognition, 53, 129-153.

Gillis, J.S. (1989). CAS: Cuestionario de ansiedad infantil. Madrid: TEA Ediciones. 
Golden, C.J. (1978). Stroop Color and Word test. A manual for Clinical and Experimental Uses. Wood Dale, Illinois: Stoelting Co.

Heaton, R.K. (1981). Wisconsin Card Sorting Test Manuel. Odessa (FL). Psychological Assessment Resource Inc.

Instituto Pontificio San Pío X (1961). Test de atención.

Kirk, S.A., McCarthy, J.J. y Kirk, W.D. (1986). Test de fluidez lexica (expresión verbal. En TEA (Eds.) ITPA, Test Illinois de Aptitudes Psicolingüísticas. Madrid: TEA.

Kovacs, M. (1992). Inventario de Depresión Infantil (CDI). Madrid: TEA Ediciones.

Logan, G.D. y Cowan, W.B. (1984). On the ability to inhibit thought and action: A theory of an act of control. Psychological Review, 91, 295-327.

Logan, G.D., Schachar, R.J. y Tannock, R. (1997). Impulsivity and inhibitory control. Psychological Science, 8, 60-64.

Loney, J. y Milich, R. (1982). Hiperactivity, Inatention and aggression in clinical practise. En: M. Wolraich y D.K. Routh (Eds.), Advances in behavioral pediatrics, 2nd ed. Greenwich: JAI Press. (pp. 113-147).

Miranda, A., Félix, V. y Ávila, C. (en prensa). Validez de las tareas neuropsicológicas. Psicología General y Aplicada.

Rey, A. (1994). Test de copia de una figura compleja. Madrid: TEA Ediciones.

Roselló, B., Amado, L. y Bo, R.M. (2000). Patrones de comorbilidad en los distintos subtipos de niños con trastorno por déficit de atención con hiperactividad. Revista de Neurología, 1, 1-12.

Rosvold, H.E., Mirsky, A., Sarason, M., Bransome, E.D. y Beck, L.H. (1956). A continuous performance test of brain damage. Journal Consulting Psychology, 20, 343-350.

Rudel, R.G., Denkla, M.B., y Broman, N. (1978) Rapid silent response to repeat target symbols by dyslexic and non-dyslexic children. Brain and Language, 6, 52-62.

Servera, M. \& Llabrés, J. (2000). EMIC (Escala Magallanes de Impulsividad Computarizada). Bilbao: Albor-Cohs.

Shallice, T. (1982). Specific impairmnets of planning. Philosophical Transactions of the Royal Society of London, B, 298, 199-209.

Siegel, L.S. y Ryan, E.B. (1989). The development of working memory in normally achieving and subtypes of learning disabled children. Child Development, 60, 973-980.

Stroop, J.P. (1935). Studies of interference in serial verbal reactions. Journal of Experimental Psychology, 18, 643-662.

Thurstone, L.L., y Yela, M. (1979) Percepción de diferencias (Caras). Madrid. TEA Ediciones. 
Wechsler, D. (1974). Wechsler Intelligence Scale for Children - Revised. The Psychological Corporation, New York. (Trad. Castellano: Escala de Inteligencia Wechsler para Niños - Revisada. WISC-R. Madrid: Tea. 1997). 